Tratamiento del suelo pélvico en varones con lesión medular incompleta: revisión sistemática

\title{
Treatment of the pelvic floor in males with incomplete spinal cord injury: a systematic review
}

https://doi.org/10.23938/ASSN.0868

\section{Hernández Rodríguez, S. Pérez- de la Cruz}

\section{RESUMEN}

Los problemas del suelo pélvico son una de las complicaciones más frecuentes en pacientes de sexo masculino con lesión medular. El objetivo de esta revisión es analizar la evidencia disponible sobre la eficacia de los distintos tratamientos que se pueden emplear en las disfunciones del suelo pélvico en varones con lesión medular incompleta. Se consultaron las bases de datos Scopus, Medline, PEDro, Web of Science, Dialnet, Pubmed y Cochrane, en castellano e inglés en los últimos veinte años. Se incluyeron quince artículos con un total de 706 pacientes varones con lesión medular incompleta. La neuromodulación y la combinación de tratamientos (electroterapia o acupuntura / electroacupuntura con cateterismo o entrenamiento de vejiga) reportan los mejores resultados en vejigas neurógenas y disfunciones eréctiles.

Palabras clave. Lesión medular. Suelo pélvico. Tratamiento conservador. Varones.

\begin{abstract}
Pelvic floor disorders are one of the most common complications in male patients with spinal cord injury The aim of this review was to analyse the available evidence on the efficacy of different treatments used for pelvic floor dysfunctions in men with incomplete spinal cord injuries. The Scopus, Medline, PEDro, Web of Science, Dialnet, Pubmed and Cochrane databases were consulted for papers in Spanish and English published in the last twenty years. Fifteen articles were included, comprising a total of 706 male patients with incomplete spinal cord injury. In male patients with neurogenic bladder and erectile dysfunction, the best results were obtained with neuromodulation and combined treatments (electrotherapy or acupuncture / electroacupuncture with catheterization or bladder training).
\end{abstract}

Keywords. Incomplete spinal cord injury. Pelvic floor. Conservative treatment. Male.
Departamento de Enfermería, Fisioterapia y Medicina. Universidad de Almería.

Recepción: 27/02/2020

Aceptación provisional: $23 / 04 / 2020$

Aceptación definitiva: 25/05/2020

\section{Correspondencia:}

Sagrario Pérez- de la Cruz

Facultad de Ciencias de la Salud

Universidad de Almería

Ctra. Sacramento, s/n

04120 La Cañada de San Urbano (Almería)

E-mail: spd205@ual.es 


\section{INTRODUCCIÓN}

La lesión medular se define como un proceso patológico de etiología traumática y no traumática, caracterizado por trastornos de la motricidad y sensibilidad corporal por debajo de la zona lesionada, que depende del nivel y complejidad de la misma ${ }^{1}$. Afecta también, en su gran mayoría, al nivel psicosocial tanto de la persona afectada como de las personas de su alrededor $^{1,2}$. Esta lesión puede ser completa, con la pérdida por debajo de la lesión de las funciones sensitivas y motoras; o incompleta, cuando aún existe inervación parcial o total por debajo del nivel lesionado.

En España, la población que sufre una lesión medular es de 12-20 personas por cada millón de habitantes al año. A nivel mundial, la incidencia oscila entre 10,4 y 83 personas por millón de habitantes/año, viéndose afectadas entre 250.000 y 500.000 personas cada año ${ }^{2}$. La mayor incidencia se sitúa actualmente entre los 30 y 40 años de edad $^{2}$.

La incidencia de lesiones de tipo traumático en varones es de 4:1 ${ }^{1-3}$; sin embargo se equilibra a 1:1 cuando las lesiones son de tipo no traumático ${ }^{2}$.

Como consecuencia de la lesión medular, los pacientes se tienen que enfrentar a una serie de secuelas que afectan a distintos niveles.

Una de ellas es la infección del tracto urinario. Para evitarla se propone un ajuste en la dieta (mayor aporte de líquidos) y realizar un cateterismo limpio e intermitente, o incluso aplicar antisépticos urinarios. En los casos más graves, se debería colocar una sonda permanente y contactar con el facultativo ${ }^{4}$. Pero el principal problema es la vejiga neurógena, una disfunción neurogénica del tracto urinario inferior provocada por la interrupción de la inervación de la vejiga, en la que puede fallar tanto el llenado de la vejiga (con contracciones durante esta fase, vejiga hiperrefléxica) como al vaciado (con ausencia de contracción, vejiga arrefléxica $)^{5}$. Otro motivo de problemas en las fases de llenado y de vaciado es la alteración del esfínter; además puede aparecer la disinergia detrusor-esfinter, que provoca una contracción del esfínter externo durante la contracción vesical, lo que produce un vaciamiento vesical incompleto ${ }^{4}$.

También existen secuelas que afectan a la función sexual, con alteraciones como la disfunción eréctil y la disfunción eyaculatoria, además de la pérdida de la sensibilidad orgásmica tanto en el hombre como en la mujer ${ }^{5}$. La disfunción eyaculatoria puede comprender, entre otros, un problema en la coordinación de los sistemas nerviosos simpático, parasimpático y somático, pudiendo encontrar un fallo en el cierre de la vejiga, hacia donde se dirige el semen (eyaculación retrógrada) o, por el contrario, que la persona afectada no muestre eyaculación alguna ${ }^{6,7}$.

Para los problemas urogenitales se conocen distintos tratamientos, como cateterización, medicamentos, vaciado de vejiga asistido, cirugía, masaje abdominal, etc. ${ }^{8}$, aunque el más conocido es la aplicación de la toxina botulínica cuyos efectos temporales obligan a administrarla varias veces ${ }^{4}$. Dentro de las técnicas físicas para mejorar las secuelas mencionadas se encuentran: la neuromodulación para el tratamiento de la disfunción miccional cónica (DMC) refractaria a tratamientos convencionales ${ }^{8}$; la acupuntura ${ }^{6,9}$; la electroacupuntura que combina acupuntura con técnicas de electroterapia ${ }^{2}$; el entrenamiento del suelo pélvico para disminuir la presión del detrusor y reducir la incontinencia ${ }^{10-12}$; las ayudas técnicas para facilitar la deambulación y estimular los movimientos peristálticos ${ }^{13}$; la estimulación eléctrica o electroterapia, con colocación de electrodos en la zona pélvi$\mathrm{ca}^{14} \mathrm{o}$ en nervios periféricos o cercanos ${ }^{15-20}$, y la estimulación epidural con colocación quirúrgica de electrodos en el espacio epidural (habitualmente lumbar) y de un generador a unos dos centímetros por debajo de la piel ${ }^{21}$.

Además de la administración oral o intravenosa de medicamentos y de la cirugía (como la prótesis de pene), existen otras terapias menos invasivas y más seguras, para el tratamiento de la disfunción sexual cuya finalidad es conseguir la eyaculación ${ }^{22,23}$, vi- 
braciones en el pene (laterales, en el dorso o en el frenillo), electroeyaculación mediante electrodos en el recto orientados hacia la próstata y la vesícula seminal, y masaje de próstata.

Dada la variedad de tratamientos, el principal objetivo de esta investigación es revisar la efectividad los distintos tipos de tratamientos empleados en las disfunciones del suelo pélvico en lesionados medulares varones.

\section{MATERIAL Y MÉTODOS}

Se realizó una búsqueda bibliográfica entre diciembre de 2018 y marzo de 2019. en las siguientes bases de datos: Scopus, Medline, PEDro, Web of Science, Dialnet, Pubmed y Cochrane, con la siguiente estrategia de búsqueda: "spinal cord injury" [Abstract] AND ("pelvic floor" [Abstract] OR bowel [Abstract] OR bladder [Abstract] OR "sexual function" [Abstract] AND physiotherapy [Abstract] AND male [Abstract].

Se incluyeron ensayos clínicos aleatorizados y no aleatorizados, revisiones bibliográficas y estudios descriptivos y de casos, centrados en suelo pélvico y lesión medular incompleta (ASIA B, C y D) en pacientes de sexo masculino, publicados en los últimos veinte años en inglés o castellano. Se excluyeron los estudios que no especificaban los procedimientos empleados en el tratamiento del suelo pélvico.

Se siguieron las recomendaciones PRISMA. Los títulos y resúmenes fueron revisados, excluyendo los artículos duplicados y los que no cumplieron los criterios de selección. Los artículos restantes fueron evaluados en más detalle aplicando los criterios de selección. Se extrajeron los siguientes datos de cada estudio: autor y fecha de publicación, tipo de estudio, número de participantes, escalas de valoración, intervención, metodología y resultados obtenidos. Los dos autores participaron de manera activa e independiente en el proceso de selección de estudios, revisión y extracción sistemática de datos; cualquier desacuerdo fue resuelto por consenso entre ambos.
La evaluación de la calidad metodológica se realizó utilizando la escala PEDRo. Esta escala tiene once ítems (ver tabla 2 en Resultados) y cada categoría se puntúa con un punto si cumple con los requisitos. Una puntuación más alta indica una mayor calidad metodológica, considerando que un estudio con un puntaje $\geq 6$ tiene un alto nivel de calidad (6-8: bueno; 9-10: excelente), y un estudio con un puntaje $\leq 5$ tiene un bajo nivel (4 - 5: aceptable; <4: pobre).

\section{RESULTADOS}

Un total de 15 estudios cumplieron los criterios de selección (Fig. 1): un metaanálisis ${ }^{9}$, cuatro estudios aleatorizados controlados (EAC) $)^{6,12,13,24}$, ocho estudios de casos $^{2,8,11,14-17,26}$ y dos estudios piloto de $\operatorname{casos}^{18,25}$. Sus características se recogen en la tabla 1.

El número total de participantes incluidos fue de 965 sujetos, de los cuales 735 eran lesionados medulares incompletos (76,2\%) y 230 completos, la mayoría varones $(76,2 \%)$. Esta revisión se centró en los participantes varones lesionados medulares incompletos $(73,2 \%)$.

Los tamaños de muestra oscilaron entre dos (varones) ${ }^{11}$ y 334 pacientes (247 varones) ${ }^{9}$. La edad de los pacientes osciló entre 8 y 76 años, con una media de 44,1 años.

\section{Calidad metodológica}

Las puntuaciones obtenidas en la escala PEDRo se muestran en la tabla 2. Oscilaron entre cuatro ${ }^{11,14,25}$ y nueve $\mathrm{e}^{24,26}$ puntos. No hubo estudios con calidad pobre $(<4)$. El 43,8\% de los estudios revisados mostró un alto nivel de calidad ( $\geq 6$ puntos), mientras que el $56,3 \%$ mostró una baja calidad (4-5 puntos). Los criterios donde se obtuvo peor puntuación en los estudios fueron los criterios 3 (la asignación fue oculta), 7 (todos los evaluadores que midieron al menos un resultado clave fueron cegados) y 9 (se presentaron resultados de todos los sujetos que recibieron tratamiento o fueron asignados al grupo control). 


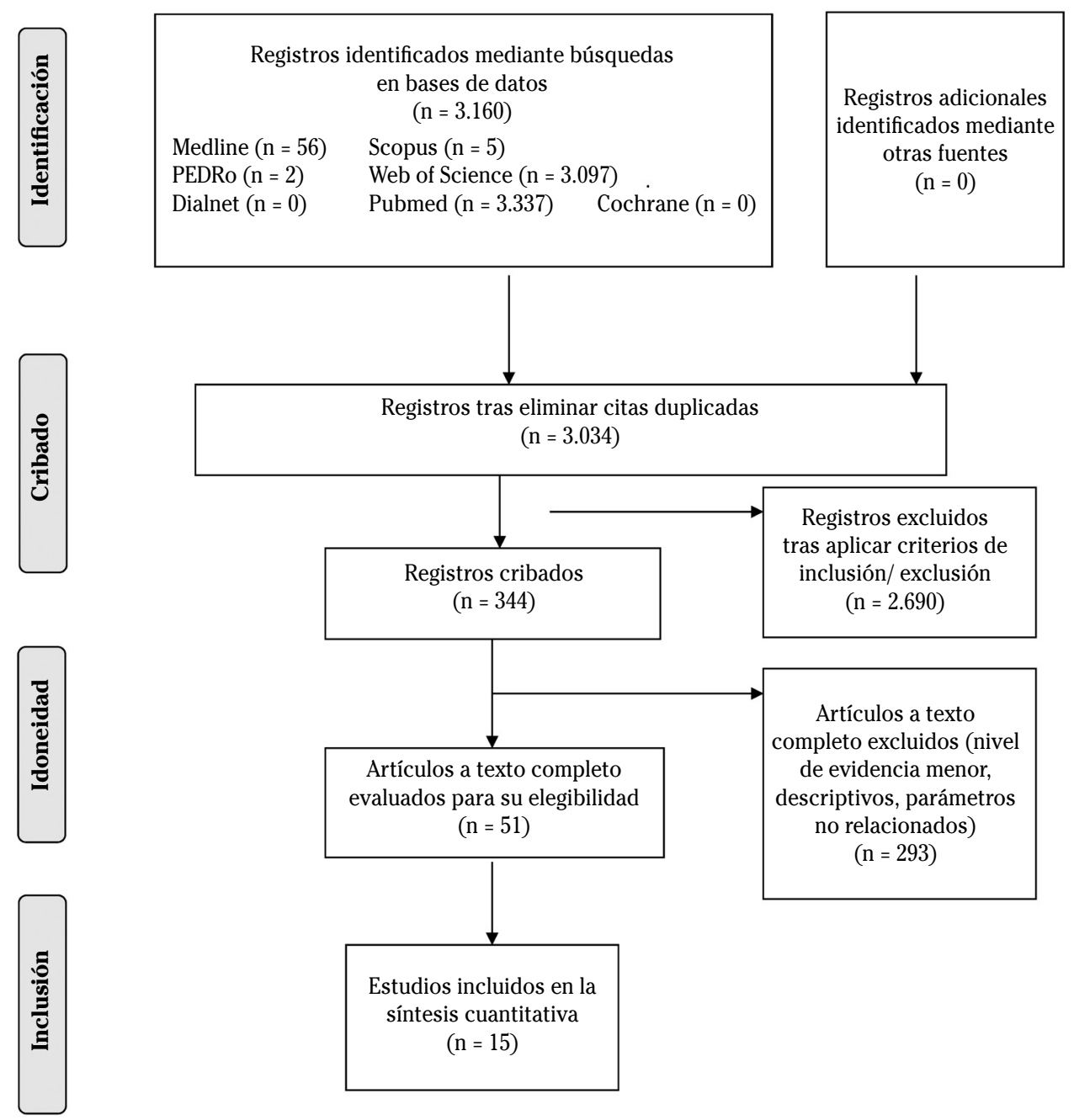

Figura 1. Flujograma PRISMA.

Tabla 1. Estudios seleccionados

\begin{tabular}{|c|c|c|c|c|c|}
\hline $\begin{array}{l}\text { Autor } \\
\text { Año }\end{array}$ & $\begin{array}{l}\text { Tipo de } \\
\text { estudio }\end{array}$ & $\begin{array}{l}\text { N, edad, lesión } \\
\text { (total y/o varones) }\end{array}$ & Intervención & $\begin{array}{c}\text { Escalas y } \\
\text { metodología de } \\
\text { valoración }\end{array}$ & Resultados \\
\hline $\begin{array}{l}\text { Liu } \\
\text { y col}^{2} \\
2013\end{array}$ & Casos & $\begin{array}{l}-\mathrm{N}=14 \\
-34 \text { años } \\
-\mathrm{N} \text { varones = } 9 \\
\quad(64,3 \%) \\
\text { - Grado: } \\
\text { A n }=1 \\
\text { B n }=6 \\
\text { C n }=2\end{array}$ & $\begin{array}{l}\text { - EA en la zona de S2- } \\
\text { S3, } 50 \text { minutos } 1 \text { vez/ } \\
\text { día, } 5 \text { días/semana y } \\
\text { luego } 3 \text { días/semana } \\
\text { hasta: } 6 \text { meses }\end{array}$ & $\begin{array}{l}\text { - Escala ASIA. } \\
\text { - Valoración/ registro } \\
\text { de frecuencia de } \\
\text { micción normal } \\
\text { - Incontinencia } \\
\text { urinaria } \\
\text { - Volumen de } \\
\text { orina residual y } \\
\text { de evacuación } \\
\text { intestinal }\end{array}$ & $\begin{array}{l}\text { - Disminuye el volumen de } \\
\text { orina residual }(\mathrm{p}<0,001) \\
\text { - Mejora la continencia urinaria } \\
\text { ( } \mathrm{p}=0,036) \text { y la motilidad/ } \\
\text { control intestinal }\end{array}$ \\
\hline
\end{tabular}




\begin{tabular}{|c|c|c|c|c|c|}
\hline $\begin{array}{l}\text { Autor } \\
\text { Año }\end{array}$ & $\begin{array}{l}\text { Tipo de } \\
\text { estudio }\end{array}$ & $\begin{array}{c}\mathrm{N}, \text { edad, lesión } \\
\text { (total y/o varones) }\end{array}$ & Intervención & $\begin{array}{c}\text { Escalas y } \\
\text { metodología de } \\
\text { valoración }\end{array}$ & Resultados \\
\hline $\begin{array}{l}\text { Xial } \\
\text { y col }^{6} \\
2014\end{array}$ & $\mathrm{ECA}$ & $\begin{array}{l}-\mathrm{N}=42 \\
-37,3 \text { años } \\
-\mathrm{N} \text { varones = } 27 \\
\quad(64,3 \%) \\
\text { - Incompleta }\end{array}$ & $\begin{array}{l}\text { - GE: EA en los puntos } \\
\text { Ren4 y Ren3, más } \\
\text { entrenamiento de } \\
\text { vejiga } \\
\text { - GC: entrenamiento } \\
\text { de vejiga }\end{array}$ & $\begin{array}{l}\text { - Medidas } \\
\text { urodinámicas } \\
\text { (capacidad de la } \\
\text { vejiga, volumen } \\
\text { residual, presión } \\
\text { rectal, presión } \\
\text { detrusor) } \\
\text { - Escala internacional } \\
\text { de síntomas de } \\
\text { próstata } \\
\end{array}$ & $\begin{array}{l}\text { - Aumenta la capacidad de la } \\
\text { vejiga }(\mathrm{p}<0,05) \\
\text { - Disminuye volumen residual, } \\
\text { presión de la vejiga, presión } \\
\text { rectal y presión del detrusor } \\
(\mathrm{p}<0,05)\end{array}$ \\
\hline $\begin{array}{l}\text { Lombardi } \\
\text { y col}^{8} \\
2011\end{array}$ & Casos & $\begin{array}{l}\text { - } \mathrm{N}=37(100 \%) \\
-18-75 \text { años } \\
\text { - Incompleta, con } \\
\text { dos disfunciones } \\
\text { pelvianas }\end{array}$ & $\begin{array}{l}\text { - Neuromodulación } \\
\text { sacra con electrodo } \\
\text { en tercer foramen } \\
\text { del sacro, y } \\
\text { generador en la } \\
\text { nalga }\end{array}$ & $\begin{array}{l}\text { - Escala ASIA. } \\
\text { - Medidas } \\
\text { urodinámicas, } \\
\text { cistometría y } \\
\text { presión del detrusor } \\
\text { - Îndice internacional } \\
\text { de la disfunción } \\
\text { eréctil }\end{array}$ & $\begin{array}{l}\text { - Aumenta la capacidad de la } \\
\text { vejiga } \\
\text { - Disminuye presión del } \\
\text { detrusor. Mejoría del intestino } \\
\text { neurógeno y de la disfunción } \\
\text { eréctil. }(\mathrm{p}<0,05)\end{array}$ \\
\hline $\begin{array}{l}\text { Wang } \\
\text { y col }^{9} \\
2016\end{array}$ & $\begin{array}{l}\text { Meta- } \\
\text { análisis }\end{array}$ & $\begin{array}{l}-\mathrm{N}=334(100 \%) \\
\mathrm{n} 1=70, \mathrm{n} 2=264 \\
-36,2 \text { años }(8-71) \\
\text { - Incompleta }\end{array}$ & $\begin{array}{l}\text { - } 1 \text { : Acupuntura } \\
\text { manual y } \\
\text { rehabilitación } \\
\text { vesical } v s \\
\text { rehabilitación } \\
\text { - 2: EA y cateterización } \\
\text { aséptica intermitente } \\
\text { vs cateterización } \\
\text { aséptica intermitente }\end{array}$ & $\begin{array}{l}\text { - Volumen de orina } \\
\text { residual post } \\
\text { vaciado } \\
\text { - Capacidad de } \\
\text { micción automática } \\
\text { - Desaparición } \\
\text { de síntomas de } \\
\text { retención urinaria } \\
\text { - SF-36 }\end{array}$ & $\begin{array}{l}\text { - No se aportan resultados de } \\
\text { calidad de vida } \\
\text { - Mejora del control de } \\
\text { retención crónica urinaria con } \\
\text { combinación de tratamientos } \\
\text { 1: Disminuye el volumen de } \\
\text { orina residual post vaciado } \\
\text { (-109,44; IC95\%: -156,53 a } \\
\text {-62,35; p< } 0,001 \text { ) } \\
\text { 2: Mejor tasa de repuesta: } 1,23 \\
\text { (IC95\%: } 1,10 \text { a } 1,38 ; p<0,001) \\
\end{array}$ \\
\hline $\begin{array}{l}\text { Vásquez } \\
\text { y col }^{11} \\
2015\end{array}$ & Casos & $\begin{array}{l}-\mathrm{N}=2(100 \%) \\
-53 \text { y } 64 \text { años } \\
\text { - Grado C con lesión } \\
\text { en T11; Grado D en } \\
\text { C3 }\end{array}$ & $\begin{array}{l}\text { Entrenamiento } \\
\text { de suelo pélvico } \\
\text { ( } 40 \text { contracciones } \\
\text { divididas en } 4 \text { sets), } \\
3 \text { veces/día por } 6 \\
\text { semanas }\end{array}$ & $\begin{array}{l}\text { - Escala ASIA } \\
\text { - Escala Oxford } \\
\text { modificada } \\
\text { - Cistometría } \\
\text { - Cuestionario de } \\
\text { incontinencia }\end{array}$ & $\begin{array}{l}\text { - Aumento de fuerza en ambos } \\
\text { sujetos } \\
\text { - Mejora de hiperactividad del } \\
\text { detrusor en grado D ( } 81 \% \\
\text { frente al } 16 \% \text { del grado C), } \\
\text { controlando la incontinencia } \\
\text { (10\% en Grado D frente a no } \\
\text { control en grado C) }\end{array}$ \\
\hline $\begin{array}{l}\text { Abdel-azim } \\
\text { y coll }^{12} \\
2015\end{array}$ & $\mathrm{ECA}$ & $\begin{aligned}-\mathrm{N}=30(100 \%) \\
\mathrm{GC}=28,1(5,4) \\
\mathrm{GE}=28.3(5,1) \\
-20-35 \text { años } \\
\text { - Incompleta por } \\
\text { encima de T12 }\end{aligned}$ & $\begin{array}{l}\text { GC: biofeedback } \\
\text { GE: TENS junto con } \\
\text { ejercicios de suelo } \\
\text { pélvico } \\
12 \text { sesiones de } 30 \text { min } \\
2 \text { veces/semana }\end{array}$ & $\begin{array}{l}\text { - Cistometría } \\
\text { - Medición de la } \\
\text { actividad muscular } \\
\text { del suelo pélvico } \\
\text { - Cuestionario del } \\
\text { índice Internacional } \\
\text { de Disfunción Eréctil } \\
\text { (IIEF-5) }\end{array}$ & $\begin{array}{l}\text { - TENS mejora disfunción } \\
\text { urinaria y eréctil, mayor } \\
\text { vaciado vesical, mejora } \\
\text { capacidad máxima de la } \\
\text { vejiga, velocidad máxima de } \\
\text { flujo ( } \mathrm{p}<0,001 \text { ) y puntuación } \\
\text { en escala IIEF-5 frente al } \\
\text { biofeedback }\end{array}$ \\
\hline $\begin{array}{l}\text { Huang } \\
\text { y col }^{13} \\
2015\end{array}$ & ECA & $\begin{array}{l}\text { - N }=24 \\
\text { GE: } 41,7 \text { (3,3) años } \\
\text { GC: } 38,4(2,25) \text { años } \\
\text { - N varones = } 16 \\
\text { (66,7\%) } \\
\text { GE: } 9(75 \%) \\
\text { GC: } 7(58,3 \%) \\
\text { - Lesiones de T8 a L2 }\end{array}$ & $\begin{array}{l}\text { GE: rehabilitación } \\
\text { asistida con un robot } \\
\text { GC: entrenamiento } \\
\text { en cinta rodante } \\
\text { soportando su propio } \\
\text { peso } \\
20 \text { minutos, cuatro } \\
\text { veces/ semana, } \\
\text { durante un mes }\end{array}$ & $\begin{array}{l}\text { - Tiempo de } \\
\text { defecación y uso de } \\
\text { enemas (dosis) }\end{array}$ & $\begin{array}{l}\text { - El grupo asistido por } \\
\text { robot disminuye periodo } \\
\text { de defecación }(\mathrm{p}<0,01) \text { y } \\
\text { aumenta el peristaltismo y la } \\
\text { función intestinal }\end{array}$ \\
\hline
\end{tabular}




\begin{tabular}{|c|c|c|c|c|c|}
\hline $\begin{array}{l}\text { Autor } \\
\text { Año }\end{array}$ & $\begin{array}{l}\text { Tipo de } \\
\text { estudio }\end{array}$ & $\begin{array}{c}\mathrm{N}, \text { edad, lesión } \\
\text { (total y/o varones) }\end{array}$ & Intervención & $\begin{array}{c}\text { Escalas y } \\
\text { metodología de } \\
\text { valoración }\end{array}$ & Resultados \\
\hline $\begin{array}{l}\text { Ishigooka } \\
\text { y col }^{14} \\
1996\end{array}$ & Casos & $\begin{array}{l}\text { - } \mathrm{N}=6(100 \%) \\
-36,8 \text { años } \\
\text { - Lesión dorsal }(50 \%), \\
\text { cervical }(33,3 \%) \\
\text { lumbar }(16,6 \%)\end{array}$ & $\begin{array}{l}\text { Estimulación eléctrica } \\
\text { a nivel del suelo } \\
\text { pélvico, } 30 \text { minutos } \\
\text { cuatro veces por día }\end{array}$ & $\begin{array}{l}\text { - Cistometría } \\
\text { - Volumen de la } \\
\text { vejiga y presión del } \\
\text { detrusor }\end{array}$ & $\begin{array}{l}\text { - Mejora de la incontinencia y } \\
\text { disminución de la presión en } \\
50 \% \text { pacientes } \\
\text { - Aumento del volumen en un } \\
\text { 83\% de ellos }\end{array}$ \\
\hline $\begin{array}{l}\text { Brose } \\
\mathrm{y} \mathrm{col}^{15} \\
2017\end{array}$ & Casos & $\begin{array}{l}-\mathrm{N}=23(100 \%) \\
\text { - } 59,8 \text { años } \\
\text { - Grado An }=1(4,3 \%), \\
\text { B n }=2(8,7 \%) \\
\text { C n }=4(17,4 \%) \\
\text { D } n=16(69,6 \%)\end{array}$ & $\begin{array}{l}\text { Electroestimulación } \\
\text { del nervio genital }\end{array}$ & $\begin{array}{l}\text { - Presión abdominal y } \\
\text { del detrusor } \\
\text { - Medidas } \\
\text { urodinámicas }\end{array}$ & $\begin{array}{l}\text { - Mejora en la capacidad de la } \\
\text { vejiga }(\mathrm{p}<0,01) \\
\text { - Disminuyen las contracciones } \\
\text { de la vejiga en un } 50 \% \text { de los } \\
\text { pacientes }\end{array}$ \\
\hline $\begin{array}{l}\text { Lee } \\
\mathrm{y} \mathrm{col}^{16} \\
2011\end{array}$ & Casos & $\begin{array}{l}-\mathrm{N}=40 \\
-44 \text { años } \\
-\mathrm{N} \text { varones = } 36(90 \%) \\
\text { - Incompleta } \mathrm{n}=14 \\
\quad(39 \%)\end{array}$ & $\begin{array}{l}\text { Electroestimulación } \\
\text { del nervio pudendo }\end{array}$ & $\begin{array}{l}\text { - Escala ASIA. } \\
\text { - Prueba cistometría } \\
\text { - Actividad del } \\
\text { detrusor }\end{array}$ & $\begin{array}{l}\text { - Descenso en las } \\
\text { contracciones y actividad del } \\
\text { detrusor } \\
\text { - Aumento de } 174,9 \mathrm{ml} \text { en el } \\
\text { volumen expulsado pre- } v s \\
\text { post- intervención }\end{array}$ \\
\hline $\begin{array}{l}\text { Zempleni } \\
\text { y col }^{17} \\
2010\end{array}$ & Casos & $\begin{array}{l}-\mathrm{N}=8 \\
\text { - Edad no disponible } \\
\text { - } \mathrm{N} \text { varones = } 4(50 \%) \\
\text { - Incompleta } \mathrm{n}=3 \\
\quad(75 \%)\end{array}$ & $\begin{array}{l}\text { Estimulación del } \\
\text { nervio pudendo, } 15 \\
\text { minutos dos veces/ } \\
\text { día }\end{array}$ & $\begin{array}{l}\text { - Uso de resonancia } \\
\text { magnética funcional. } \\
\text { - Prueba cistometría } \\
\text { (llenado y vaciado } \\
\text { de vejiga) }\end{array}$ & $\begin{array}{l}\text { - Se producen cambios a nivel } \\
\text { del encéfalo: aumento de } \\
\text { sensación de la vejiga y un } \\
\text { vaciado voluntario excepto } \\
\text { en un paciente con lesión } \\
\text { incompleta }\end{array}$ \\
\hline $\begin{array}{l}\text { Lee } \\
\mathrm{y} \mathrm{col}^{18} \\
2012\end{array}$ & $\begin{array}{l}\text { Casos } \\
\text { piloto }\end{array}$ & $\begin{array}{l}-\mathrm{N}=6(100 \%) \\
-44,5 \text { años } \\
-\mathrm{N} \text { varones = } 27 \\
\quad(64,3 \%) \\
\text { - Entre C5 y T10 }\end{array}$ & $\begin{array}{l}\text { Estimulación eléctrica } \\
\text { del nervio dorsal del } \\
\text { pene, 1-3 veces por día } \\
\text { durante 14-28 días }\end{array}$ & $\begin{array}{l}\text { - Prueba cistometría } \\
\text { - Escala ASIA } \\
\text { - Medidas de presión } \\
\text { - Reflujo vesicouretral }\end{array}$ & $\begin{array}{l}\text { - Disminuye la actividad del } \\
\text { detrusor } \\
\text { - Aumento del volumen y la } \\
\text { elasticidad de la vejiga }\end{array}$ \\
\hline $\begin{array}{l}\text { McGee } \\
\mathrm{y} \mathrm{col}^{20} \\
2015\end{array}$ & $\begin{array}{l}\text { Revisión } \\
\text { narrativa }\end{array}$ & $\begin{array}{l}175 \text { pacientes con } \\
\text { diagnóstico de } \\
\text { lesión medular } \\
\text { (Desde ASIA A hasta } \\
\text { ASIA C). }\end{array}$ & $\begin{array}{l}\text { Electroestimulación } \\
\text { percutánea a nivel } \\
\text { de los nervios tibial } \\
\text { posterior, y pudendo. }\end{array}$ & $\begin{array}{l}\text { - Estudios } \\
\text { urodinámicos } \\
\text { - Presión del detrusor }\end{array}$ & $\begin{array}{l}\text { - Aumenta el volumen de la } \\
\text { vejiga } \\
\text { - Disminuyen las contracciones } \\
\text { de la vejiga y presión del } \\
\text { detrusor } \\
\text { - Mejoría de la incontinencia } \\
\text { urinaria }\end{array}$ \\
\hline $\begin{array}{l}\mathrm{Gu} \\
\mathrm{y} \mathrm{col}^{24} \\
2015\end{array}$ & ECA & $\begin{array}{l}-\mathrm{N}=107 \\
\text { - 35,7 años (20-57) } \\
\text { - Grado: } \\
\text { B n }=37(34,6 \%) \\
\text { C n }=53(49,5 \%) \\
\text { D n }=17(15,9 \%) \\
\text { - N varones }=80 \\
(74,8 \%)\end{array}$ & $\begin{array}{l}\text { GC: CAI } \\
\text { GE1: CAI y EA } \\
\text { GE2: CAI y acupuntura } \\
\text { simulada }\end{array}$ & $\begin{array}{l}\text { - Escala ASIA } \\
\text { - Volumen de orina } \\
\text { residual y volumen } \\
\text { de vaciado }\end{array}$ & $\begin{array}{l}\text { - Disminución del volumen } \\
\text { de orina residual y de la } \\
\text { frecuencia de cateterización } \\
(\mathrm{p}<0,001) \\
\text { - Aumento del volumen del } \\
\text { vaciado ( } \mathrm{p}<0,001) \\
\text { - Mejoría de la contracción de } \\
\text { la vejiga }\end{array}$ \\
\hline $\begin{array}{l}\text { Ojha } \\
\mathrm{y} \mathrm{col}^{25} \\
2014\end{array}$ & $\begin{array}{l}\text { Casos } \\
\text { piloto }\end{array}$ & $\begin{array}{l}-\mathrm{N}=15(100 \%) \\
-35,8 \text { años } \\
\text { - Incompleta } \mathrm{n}=10 \\
\quad(67 \%) ; \\
\text { Grado: } \\
\text { An }=5(50 \%) \\
\text { B n }=1(10 \%) \\
\text { C n }=3(30 \%) \\
\text { Dn }=1(10 \%)\end{array}$ & $\begin{array}{l}\text { Neuromodulación } \\
\text { de los nervios tibial } \\
\text { posterior y dorsal del } \\
\text { pene por estimulación } \\
\text { eléctrica superficial }\end{array}$ & $\begin{array}{l}\text { - Volumen, número } \\
\text { de fugas, presión } \\
\text { máxima y capacidad } \\
\text { cistométrica } \\
\text { - Cuestionario de } \\
\text { calidad de vida } \\
\text { - Escala ASIA } \\
\text { - Estándares } \\
\text { internacionales } \\
\text { de clasificación } \\
\text { neurológica de la } \\
\text { lesión medular }\end{array}$ & $\begin{array}{l}\text { - Descenso de la presión } \\
\text { máxima y aumento del } \\
\text { volumen con estimulación del } \\
\text { tibial posterior }(\mathrm{p}=0,02) v s \\
\text { estimulación del nervio dorsal } \\
\text { del pene }(\mathrm{p}=0,062) \\
\text { - Capacidad cistométrica sin } \\
\text { variación en ambos grupos }\end{array}$ \\
\hline
\end{tabular}




\begin{tabular}{|c|c|c|c|c|c|}
\hline $\begin{array}{l}\text { Autor } \\
\text { Año }\end{array}$ & $\begin{array}{l}\text { Tipo de } \\
\text { estudio }\end{array}$ & $\begin{array}{c}\mathrm{N}, \text { edad, lesión } \\
\text { (total y/o varones) }\end{array}$ & Intervención & $\begin{array}{c}\text { Escalas y } \\
\text { metodología de } \\
\text { valoración }\end{array}$ & Resultados \\
\hline $\begin{array}{l}\text { Rasmussen } \\
\text { y coll }^{26} \\
2015\end{array}$ & Casos & $\begin{array}{l}-\mathrm{N}=277 \\
\text { - } 49 \text { años } \\
\text { - Grado: } \\
\text { A n }=234(84 \%) \\
\text { B n }=38(14 \%) \\
\text { C n }=5(2 \%) \\
\text { - N varones = } 145 \\
\quad(52,3 \%)\end{array}$ & $\begin{array}{l}\text { Neuromodulación por } \\
\text { estimulación de la raíz } \\
\text { anterior sacra }\end{array}$ & $\begin{array}{l}\text { - Escala visual } \\
\text { análoga del dolor } \\
\text { (EVA) } \\
\text { - Escala ASIA } \\
\text { - Neurogenic bowel } \\
\text { dysfunction score } \\
\text { - Escala de } \\
\text { incontinencia fecal } \\
\text { - Escala de } \\
\text { estreñimiento de } \\
\text { Cleveland }\end{array}$ & $\begin{array}{l}\text { - Disminución del estreñimiento } \\
\text { y la incontinencia fecal } \\
\text { ( }<<0,001) \\
\text { - Mejora la puntuación en } \\
\text { Escala de Cleveland }(\mathrm{p}<0,001) \\
\text { - Disminuye la percepción del } \\
\text { dolor (descenso de } 6 \text { a } 4 \text { en } \\
\text { EVA) }\end{array}$ \\
\hline
\end{tabular}

EA: electroacupuntura; GC: grupo control; GE: grupo estudio; ECA: estudio aleatorizado controlado; TENS: Electroestimulación nerviosa transcutánea; CAI: cateterización aséptica intermitente.

Tabla 2. Puntuaciones obtenidas tras el análisis metodológico según la escala PEDRo

\begin{tabular}{|c|c|c|c|c|c|c|c|c|c|c|c|c|}
\hline Autor (año) & 1 & 2 & 3 & 4 & 5 & 6 & 7 & 8 & 9 & 10 & 11 & Puntuación total \\
\hline Ishigooka y col $^{14}(1996)$ & $\sqrt{ }$ & & & $\sqrt{ }$ & & & & & & $\sqrt{ }$ & $\sqrt{ }$ & 4 \\
\hline Zempleni y col' ${ }^{17}(2010)$ & $\sqrt{ }$ & & & $\sqrt{ }$ & $\sqrt{ }$ & & & & & $\sqrt{ }$ & $\sqrt{ }$ & 5 \\
\hline Lombardi y col ${ }^{8}$ (2011) & $\sqrt{ }$ & & & $\sqrt{ }$ & $\sqrt{ }$ & & & & & $\sqrt{ }$ & $\sqrt{ }$ & 5 \\
\hline Lee $\mathrm{y} \mathrm{col}^{16}(2011)$ & $\sqrt{ }$ & & & $\sqrt{ }$ & & & & & & $\sqrt{ }$ & $\sqrt{ }$ & 4 \\
\hline Lee y $\operatorname{col}^{18}(2012)$ & $\sqrt{ }$ & & & $\sqrt{ }$ & $\sqrt{ }$ & & & & & $\sqrt{ }$ & $\sqrt{ }$ & 5 \\
\hline Liu y col² (2013) & $\sqrt{ }$ & & & $\sqrt{ }$ & $\sqrt{ }$ & & $\sqrt{ }$ & & $\sqrt{ }$ & $\sqrt{ }$ & $\sqrt{ }$ & 7 \\
\hline Xial y $\operatorname{col}^{6}(2014)$ & $\sqrt{ }$ & $\sqrt{ }$ & & $\sqrt{ }$ & $\sqrt{ }$ & & & & & $\sqrt{ }$ & $\sqrt{ }$ & 6 \\
\hline Ojha y col${ }^{25}(2014)$ & & & & $\sqrt{ }$ & $\sqrt{ }$ & & & & & $\sqrt{ }$ & $\sqrt{ }$ & 4 \\
\hline Vásquez y col ${ }^{11}$ (2015) & $\sqrt{ }$ & & & $\sqrt{ }$ & & & & & & $\sqrt{ }$ & $\sqrt{ }$ & 4 \\
\hline Abdel-azim y col' ${ }^{12}$ (2015) & & $\sqrt{ }$ & & $\sqrt{ }$ & $\sqrt{ }$ & & & & & $\sqrt{ }$ & $\sqrt{ }$ & 5 \\
\hline Huang y col ${ }^{13}(2015)$ & $\sqrt{ }$ & $\sqrt{ }$ & & $\sqrt{ }$ & $\sqrt{ }$ & & $\sqrt{ }$ & $\sqrt{ }$ & & $\sqrt{ }$ & $\sqrt{ }$ & 8 \\
\hline Gu y $\operatorname{col}^{24}(2015)$ & $\sqrt{ }$ & $\sqrt{ }$ & & $\sqrt{ }$ & $\sqrt{ }$ & $\sqrt{ }$ & & $\sqrt{ }$ & $\sqrt{ }$ & $\sqrt{ }$ & $\sqrt{ }$ & 9 \\
\hline Rasmussen y col ${ }^{26}$ (2015) & $\sqrt{ }$ & $\sqrt{ }$ & & $\sqrt{ }$ & $\sqrt{ }$ & & $\sqrt{ }$ & $\sqrt{ }$ & $\sqrt{ }$ & $\sqrt{ }$ & $\sqrt{ }$ & 9 \\
\hline Wxang y $\operatorname{col}^{9}(2016)$ & & & & $\sqrt{ }$ & & & $\sqrt{ }$ & $\sqrt{ }$ & $\sqrt{ }$ & $\sqrt{ }$ & $\sqrt{ }$ & 6 \\
\hline Brose y coll ${ }^{15}(2017)$ & $\sqrt{ }$ & & & $\sqrt{ }$ & $\sqrt{ }$ & & & $\sqrt{ }$ & & $\sqrt{ }$ & $\sqrt{ }$ & 6 \\
\hline
\end{tabular}

$\sqrt{ }$ : el estudio cumplió con los criterios de la escala PEDRo: 1) los criterios de elección fueron especificados; 2) los sujetos fueron asignados al azar a los grupos; 3 ) la asignación fue oculta; 4) los grupos fueron similares al inicio en relación a los indicadores de pronóstico más importantes; 5) todos los sujetos fueron cegados; 6) todos los terapeutas que administraron la terapia fueron cegados; 7) todos los evaluadores que midieron al menos un resultado clave fueron cegados; 8) las medidas de al menos uno de los resultados clave fueron obtenidas de más del $85 \%$ de los sujetos inicialmente asignados a los grupos; 9) se presentaron resultados de todos los sujetos que recibieron tratamiento o fueron asignados al grupo control, o cuando esto no pudo ser, los datos para al menos un resultado clave fueron analizados por "intención de tratar"; 10) los resultados de comparaciones estadísticas entre grupos fueron informados para al menos un resultado clave; 11) el estudio proporciona medidas puntuales y de variabilidad para al menos un resultado clave.

\section{Neuromodulación}

Es una técnica que requiere la implantación de un electrodo. Este puede ser profundo $^{8,26}$ (en el tercer agujero sacro posterior, con un generador externo de impulsos permanente en la nalga, utilizando una frecuencia de entre 5 y $20 \mathrm{~Hz}^{8}$ ) o superficial ${ }^{25}$, en nervios periféricos como el tibial posterior y el dorsal del pene.

Lombardi y $\mathrm{col}^{8}$ y Rasmussen y $\mathrm{col}^{26}$ obtuvieron muy buenos resultados con la 
neuromodulación profunda en la recuperación tanto de la vejiga como del intestino neurógenos (disminución de 6 puntos en la media de disfunción neurogénica intestinal, y mejora en la puntuación promedio en la escala de estreñimiento de Cleveland) y en la percepción de dolor (puntuación VAS disminuyó de 6 a 4$)^{26}$.

Ojha y $\mathrm{col}^{25}$ aplicaron la neuromodulación de manera superficial en nervios periféricos durante cuatro semanas, obteniéndose una mejora significativa con la estimulación del nervio tibial posterior. La estimulación del nervio dorsal del pene logró un descenso de la presión máxima y un aumento del volumen no significativos. Los datos de capacidad cistométrica no fueron estadísticamente significativos, aunque se observó una mejoría en el diario miccional.

\section{Electroacupuntura}

La acupuntura es una técnica que consiste en introducir agujas en determinados puntos del cuerpo, de forma manual o junto con una corriente eléctrica, como Liu y $\mathrm{col}^{2}$. Estos autores lograron diez de sus quince pacientes (67\%) adquirieran la habilidad del vaciado voluntario de la vejiga sin necesidad de aplicar métodos complementarios, logrando disminuir el volumen de orina residual, mejorar la continencia urinaria y la motilidad/control intestinal.

\section{Terapia física}

Los dos participantes del estudio de Vásquez $\mathrm{y} \mathrm{col}^{11}$ realizaron ejercicios de suelo pélvico; ambos mejoraron en fuerza, mientras que la hiperactividad del músculo detrusor solo mejoró en el paciente con Grado D de afectación.

El entrenamiento con biofeedback consiste en la realización de ejercicios asistidos por un instrumento que traduce la contracción muscular en una señal gráfica, acústica o ambas que permite visualizar y ser consciente del grado de contracción muscular alcanzado, para poderlo controlar y modificar de forma voluntaria. Los pa- cientes del estudio de Huang y col ${ }^{13}$ mejoraron no solo la musculatura, sino también los problemas urinarios y de disfunción eréctil gracias al biofeedback. Además, la marcha diaria facilitada por ayudas técnicas disminuyó el periodo de defecación y aumentó el peristaltismo, mejorando la función intestinal.

\section{Electroestimulación}

Las técnicas de electroterapia se emplearon en seis estudios para tratar las disfunciones del suelo pélvico ${ }^{12,14-18}$.

La colocación de electrodos de alambre fino puede ser en la zona pélvica, a nivel del esfínter externo de la uretra como en el estudio de Ishigookal y $\mathrm{col}^{14}$, logrando reducir la incontinencia urinaria en solo un $50 \%$ de los pacientes atendidos. Cuando la electroestimulación se aplica no directamente en el periné sino en nervios periféricos a la zona $^{15,16,18}$, hasta el $90 \%$ de pacientes mejoró de sus síntomas. Los pacientes del estudio de Abdel-azim y col ${ }^{12}$ recibieron electroterapia mediante dos electrodos de superficie colocados en la piel sobre el segmento $\mathrm{S} 3$, y mejoraron su incontinencia respecto al grupo con ejercicios de biofeedback del suelo pélvico ${ }^{12}$. Ambos grupos mejoraron el volumen de la vejiga, más el grupo de electroterapia.

\section{Terapias combinadas}

Wang y $\mathrm{col}^{9}$ realizaron un metaanálisis de EAC que combinaron acupuntura con otras técnicas. La combinación de acupuntura manual con entrenamiento del suelo pélvico mostró resultados significativamente mejores en la disminución del volumen de orina residual que con solo el entrenamiento Igualmente, la combinación de electroacupuntura y cateterismo aséptico intermitente mejoró significativamente la tasa de respuesta respecto a solo cateterismo. No se informaron eventos adversos graves durante las intervenciones y ni a posteriori.

La electroacupuntura se ha combinado con otras técnicas, como en los estudios de 
Xial y $\mathrm{col}^{6}$ (con entrenamiento de la vejiga) y de $\mathrm{Gu} \mathrm{y} \mathrm{col}^{24}$ (con cateterización aséptica intermitente), observándose una disminución significativa de volumen residual de orina, presión vesical, presión rectal y presión del detrusor respecto a solo cateterismo.

\section{DISCUSIÓN}

Las técnicas utilizadas para el tratamiento del suelo pélvico en el lesionado medular son muy variadas.

La neuromodulación sacra en pacientes lesionados incompletos ha demostrado mejorar tanto la vejiga neurógena, siendo capaz de vaciarla a voluntad, como el intestino neurógeno ${ }^{8,26}$ e incluso la disfunción eréctil $^{8}$. También hubo mejoría en la modulación del control simpático de la vejiga, la uretra, el intestino distal, el canal anal y la emisión seminal. La supresión de la disreflexia autonómica implicó asimismo la modulación de las vías simpáticas torácicas y lumbares hacia los vasos sanguíneos y el corazón ${ }^{8}$. Sin embargo, las mejoras que se observan son a corto plazo, ya que a largo plazo aumenta el riesgo de perder los beneficios adquiridos debido a la pérdida de sensibilidad en la zona perineal, la cual lleva a una falta de relajación de esta zona durante el vaciado de la vejiga ${ }^{6}$. Además, se pueden observar limitaciones y efectos secundarios (necesidad de nuevos implantes, dolor o hematomas) ${ }^{26}$.

La utilización de la neuromodulación superficial de los nervios tibial posterior y dorsal del pene ha logrado una mejoría a nivel urinario, disminuyendo la hiperactividad del músculo detrusor por reducción de su espasticidad. Esta mejoría se debe a que estos nervios comparten el mismo nivel de raíz que la vejiga (S2-S4) ${ }^{25}$. Los estudios que aplicaron la neuromodulación de manera superficial en nervios periféricos obtuvieron buenos resultados, especialmente en el nervio tibial posterior, con un proceso mucho menos invasivo que la estimulación percutánea ${ }^{20,25}$.

Otra opción de la neuromodulación es la estimulación sacra de las raíces anterio- res, la cual mejora principalmente la vejiga neurógena aunque, debido al nivel de raíz al que se aplica, también se observa mejoría en el esfínter anal y en la zona colorrectal distal, si se encontraran afectadas. La forma de actuación es parecida a la neuromodulación de la tercera foramina: se colocan los electrodos y estos son controlados por un aparato externo ${ }^{25}$. Es uno de los pocos métodos cuyo objetivo es tratar varios órganos afectados. Su eficacia se ve demostrada en que, a la hora de vaciar sus intestinos, los pacientes lesionados medulares incompletos son más independientes del uso de supositorios, enemas, evacuación digital, etc., ya que aumenta los movimientos peristálticos del colon, haciendo más fácil la defecación (no siendo así en lesionados completos $)^{27}$.

La electroacupuntura supone una técnica sin altos riesgos secundarios, aunque habría que tener en cuenta los distintos puntos anatómicos donde habría que aplicarla ${ }^{9}$. Se puede considerar como un tipo de neuromodulación de un carácter menos invasivo y sin efectos secundarios, ya que no necesita de medidas quirúrgicas. Liu y $\mathrm{col}^{2}$ lograron que dos tercios de los pacientes a los que se les aplicó electroacupuntura adquirieran un mayor control del vaciado voluntario de la vejiga. Distintos estudio ${ }^{6,9,24}$ han permitido apreciar cómo, combinada con otros tratamientos (ejercicios de rehabilitación vesical o cateterización aséptica intermitente) se obtienen mejores resultados que aplicando dichos tratamientos de forma aislada.

Cuando se plantea el uso de electroterapia en este tipo de pacientes, una de las opciones es implantar un electrodo en el suelo pélvico, en el esfínter externo de la uretra. Con este método puede desaparecer la incontinencia urinaria ${ }^{12,14-18}$, aunque hay algunos casos en los que no se ha observado ninguna mejoría, lo cual puede deberse al tipo de incontinencia que se trate ${ }^{14}$. Además, tiene varios efectos adversos, como la necesidad de cirugía para implantar el electrodo o cada vez que este se desplace ${ }^{14}$.

Se han propuesto otros lugares para la colocación de los electrodos y restaurar así la función de la vejiga, ya que hay estudios 
que afirman tener una menor tasa de éxito al colocar el electrodo directamente en las paredes de la vejiga ${ }^{14}$. La estimulación de nervios periféricos, como es el caso del nervio tibial, ofrece un tratamiento menos invasivo y de gran efectividad en personas con lesión medular incompleta ${ }^{20}$. La estimulación del nervio pudendo puede realizarse de manera transcutánea o por una pequeña incisión, y puede inhibir la vejiga o, por el contrario, estimularla ${ }^{16,17}$. También se puede estimular el nervio dorsal del pene, utilizando electrodos más pequeños colocados en la parte dorsal del mismo, obteniendo los mismos resultados que con la activación del nervio pudendo ${ }^{16,20}$. Incluso la estimulación de los nervios genitales mejora e incrementa la capacidad de la vejiga, disminuyendo las contracciones involuntarias de esta ${ }^{15}$. Pero, a día de hoy, se considera una opción poco viable debido a que es una técnica altamente invasiva ${ }^{20}$.

Como se ha expuesto con anterioridad, los mejores resultados se han obtenido en los estudios que han realizado terapia combinada, de acupuntura y entrenamiento del suelo pélvico ${ }^{9}$, y de electroacupuntura con entrenamiento de la vejiga ${ }^{6}$ y cateterización intermitente ${ }^{24}$

Además de las terapias mencionadas compatibles con la fisioterapia, a lo largo de la revisión se ha hablado sobre el tratamiento de estos problemas mediante otras técnicas muy eficaces, como son la toxina botulínica y los distintos métodos quirúrgicos, como la implantación de aparatos artificiales que simulan al sistema nervioso somático central, que, entre otras cosas, permite el vaciado de la vejiga. A pesar de que se aprecian muy buenos resultados, es una técnica bastante invasiva, y a la que no todo el mundo puede recurrir ${ }^{27}$. También se están viendo buenos resultados en las terapias regenerativas, gracias al uso de células provenientes del bulbo olfatorio ${ }^{28} \mathrm{o}$ de células mesenquimales de la médula ósea del propio paciente, acelerando el proceso de regeneración de los nervios dañados ${ }^{29}$.

Las limitaciones de esta revisión son una escasez de estudios publicados sobre este tema, algunos con un limitado el tiempo de aplicación ${ }^{12,13}$ y otros con un escaso núme- ro de pacientes ${ }^{11}$. Ninguno de los estudios seleccionados ha sido desarrollado en un contexto socio-cultural similar, por lo cual sería interesante también contar con estudios sobre esta temática en nuestro país para así poder detectar posibles características propias de la población española. Además, se desconocen otros factores como los aspectos sociales de esta patología y su influencia en la personalidad de los pacientes.

En conclusión, la mayoría de los estudios analizados indican que existen varias técnicas eficaces para el tratamiento de la disfunción del suelo pélvico en lesionados medulares de sexo masculino. Las terapias combinadas (acupuntura y electroacupuntura con entrenamiento del suelo pélvico) y las terapias eléctricas con biofeedback son las preferentes para el tratamiento del suelo pélvico, porque son poco invasivas y permiten obtener efectos duraderos y no tan costosos.

Para poder asegurar un mayor índice de recuperación en este tipo de pacientes sería recomendable la realización de nuevos estudios con buena calidad metodológica que aporten evidencia científica de la eficacia de las técnicas competentes para los profesionales sanitarios en el tratamiento del suelo pélvico en lesionados medulares varones.

\section{BIBLIOGRAFÍA}

1. Henao-Lema C, Pérez-Parra J. Lesiones medulares y discapacidad: Revisión bibliográfica. Aquichan 2010; 10: 157-172. https://doi. org/10.5294/aqui.2010.10.2.5

2. Liu Z, Wang W, Wu J, Zhou K, Liu B. Electroacupuncture improves bladder and bowel function in patients with traumatic spinal cord injury: results from a prospective observational study. Evidence-Based Complementary and Alternative Medicine 2013; 2013: 1-9. https://doi.org/10.1155/2013/543174

3. National Spinal Cord Injury Statistical Center. Lesión de la médula espinal Referencia rápida de datos y cifras. Birmingham, $\mathrm{AL}$ : University of Alabama, 2016. https://www. nscisc.uab.edu/public_pages/FactsFiguresArchives/Facts\%20and\%20Figures-Spanish/ 
SCI\%20Facts\%20and\%20Figures\%20at $\% 20$ a\%20Glance\%202016-Spanish.pdf

4. Strasburger lona K, Hernández Porras Y, BarQuín SANTOS E. Lesión medular: Guía para el manejo integral del paciente con LM crónica. Madrid: ASPAYM Madrid, 2013; 82. https:// www.aspaymmadrid.org/wp-content/ uploads/2018/05/guia-manejo-integral-2013. pdf

5. Benevento BT, SiPSKI ML. Neurogenic bladder, neurogenic bowel, and sexual dysfunction in people with spinal cord injury. Phys Ther 2002; 82: 601-612. https://doi.org/10.1093/ $\mathrm{ptj} / 82.6 .601$

6. XIa L, Fan F, Tang A, Ye W. Effects of electroacupuncture combined with bladder training on the bladder function of patients with neurogenic bladder after spinal cord injury. Int $\mathrm{J}$ Clin Exp Med 2014; 7: 1344-1348.

7. Huang M, Chen H, Jiang C, Xie K, Tang P, Ou R et al. Effects of botulinum toxin a injections in spinal cord injury patients with detrusor overactivity and detrusor sphincter dyssynergia. J Rehabil Med 2016; 48: 683-687. https://doi.org/10.2340/16501977-2132

8. Lombardi G, Nelli F, Mencarini M, Del Popolo G. Clinical concomitant benefits on pelvic floor dysfunctions after sacral neuromodulation in patients with incomplete spinal cord injury. Spinal Cord 2011; 49: 629-636. https:// doi.org/10.1038/sc.2010.176

9. Wang J, Zhai Y, Wu J, Zhao S, Zhou J, Liu Z. Acupuncture for chronic urinary retention due to spinal cord injury: a systematic review. J Evid Based Complementary Altern Med 2016; 2016: 1-9. https://doi. org/10.1155/2016/9245186

10. Masterson ta, Masterson JM, Azzinaro J, ManDERSOn L, Swain S, Ramasamy R. Comprehensive pelvic floor physical therapy program for men with idiopathic chronic pelvic pain syndrome: a prospective study. Transl Androl Urol. 2017; 6: 910-915. https://doi. org/10.21037/tau.2017.08.17

11. Vásquez N, Knight S, Susser J, Gall A, Ellaway $\mathrm{P}$, Craggs M. Pelvic floor muscle training in spinal cord injury and its impact on neurogenic detrusor over-activity and incontinence. Spinal Cord 2015; 53: 887-889. https://doi. org/10.1038/sc.2015.121

12. Abdel-Azim MS, Mourad HS, El Gohary AM. Efficacy of transcutaneous electrical nerve stimulation versus biofeedback training on bladder and erectile dysfunction in patients with spinal cord injury. Egypt J Neurol Psychiatry Neurosurg 2015; 52: 194-200. https://doi.org/10.4103/1110-1083.162044
13. Huang Q, Yu L, Gu R, Zhou Y, Hu C. Effects of robot training on bowel function in patients with spinal cord injury. J Phys Ther Sci 2015; 27: 1377-1378. https://doi.org/10.1589/ jpts.27.1377

14. Ishigooka M, Hashimotol T, Hayami S, SuzuKil $Y$, NAKADAL T. Electrical pelvic floor stimulation: a possible alternative treatment for reflex urinary incontinence in patients with spinal cord injury. Spinal Cord 1996; 411-415. https://doi.org/10.1038/sc.1996.73

15. Brose S, Bourbeau D, Gustafson K. Genital nerve stimulation is tolerable and effective for bladder inhibition in sensate individuals with incomplete SCI. J Spinal Cord Med 2017; 10: 1-8. https://doi.org/10.1080/10790268.201 7.1279817

16. LeE Y, Kim JM, IM HT, LeE K, Kim SH, Hur DM. Semiconditional electrical stimulation of pudendal nerve afferents stimulation to manage neurogenic detrusor overactivity in patients with spinal cord injury. Ann Rehabil Med 2011; 35: 605-612. https://doi. org/10.5535/arm.2011.35.5.605

17. Zempleni M, Michels L, Mehnert U, Schurch B, Koluis S. Cortical substrate of bladder control in SCI and the effect of peripheral pudendal stimulation. Neuroimage 2010; 49: 2983-2994. https://doi.org/10.1016/j.neuroimage.2009.10.064

18. LeE Y, Кim S, Кiм JM, Im HT, Сho IS, LeE KW. The effect of semi conditional dorsal penile nerve electrical stimulation on capacity and compliance of the bladder with deformity in spinal cord injury patients : a pilot study. Spinal Cord 2012: 289-293. https://doi. org/10.1038/sc.2011.141

19. Bourbeau DJ, Creasey GH, Sidik S, Brose SW, GusTAFSON KJ. Genital nerve stimulation increases bladder capacity after SCI: a meta-analysis. J Spinal Cord Med 2017; 15: 1-9. https://doi.org /10.1080/10790268.2017.1281372

20. McGee MJ, Amundsen CL, GriLl WM. Electrical stimulation for the treatment of lower urinary tract dysfunction after spinal cord injury. J Spinal Cord Med 2015; 38: 135-146. https:// doi.org/10.1179/2045772314y.0000000299

21. Pettigrew R, Heetderks W, Kelley C, Peng G, KROSNICK S, JAKEMAN L et al. Epidural spinal stimulation to improve bladder, bowel, and sexual function in individuals with spinal cord injuries: a framework for clinical research. IEEE Trans Biomed Eng 2017; 64: 253-262. https://doi.org/10.1109/tbme.2016.2637301

22. Ibrahim E, LynNe C, BracketT N. Male fertility following spinal cord injury: an update. Andrology 2015; 4: 13-26. https://doi.org/10.1111/ andr.12119 
23. Trofimenko V, Hotaling JM. Fertility treatment in spinal cord injury and other neurologic disease. Transl Androl Urol 2016; 5: 102116. https://doi.org/10.3978/j.issn.22234683.2015.12.10

24. Gu X, Wang J, Yu P, Li J, Yao Y, Fu J et al. Effects of electroacupuncture combined with clean intermittent catheterization on urinary retention after spinal cord injury: a single blind randomized controlled clinical trial. Int J Clin Exp Med 2015; 8: 19757-19763.

25. Ojha R, George J, Chandy B, Tharion G, DevasaHAYAM S. Neuromodulation by surface electrical stimulation of peripheral nerves for reduction of detrusor overactivity in patients with spinal cord injury: a pilot study. J Spinal Cord Med 2014; 38: 207-213. https://doi.org/1 0.1179/2045772313y.0000000175

26. Rasmussen MM, Kutzenberger J, Krogh K, Zepke F, Bodin C, Domurath B et al. Sacral anterior root stimulation improves bowel function in subjects with spinal cord injury. Spinal Cord 2015; 53: 297-301. https://doi.org/10.1038/ sc. 2015.2

27. XIAO CG. Xiao procedure for neurogenic bladder in spinal cord injury and spina bifida. Curr Bladder Dysfunct Rep 2012; 7: 83-87. https://doi.org/10.1007/s11884-012-0130-2

28. Ramón-Cueto A, Nieto-SAmpedro M. Glial. cells from adult rat olfactory bulb: immunocytochemical properties of pure cultures of ensheathing cells. Neuroscience 1992; 47: 213-220. https://doi.org/10.1016/03064522(92)90134-n

29. Vaquero J, Zurita, M Rico, Bonilla C, Aguayo C, Montilla J et al. An approach to personalized cell therapy in chronic complete paraplegia: The Puerta de Hierro phase I/II clinical trial. Cytotherapy 2016; 18: 1025-1036. https://doi. org/10.1016/j.jcyt.2016.05.003 